\title{
Erratum to: Giovanni Battista Morgagni: facial reconstruction by virtual anthropology
}

\author{
Emanuela Gualdi-Russo $^{1,2} \cdot$ Luciana Zaccagni $^{1,2} \cdot$ Valentina Russo $^{1,2}$
}

Published online: 8 February 2016

(c) Springer Science+Business Media New York 2016

Erratum to: Forensic Sci Med Pathol (2015)

11:222-227

DOI 10.1007/s12024-015-9665-9

A revised version of "Acknowledgments" section is presented here to update the text in the originally published version.
It should read as given below:

Acknowledgments The authors would like to thank Simonetta Zonari for helping in technical procedures and Alia Binazzi for her linguistic revision of this paper. Thanks are also expressed to Prof. G. Thiene and his collaborators in University of Padua (Italy) for access to skeletal remains.

The online version of the original article can be found under doi:10.1007/s12024-015-9665-9.

Luciana Zaccagni

luciana.zaccagni@unife.it

1 Department of Biomedical and Specialty Surgical Sciences,

University of Ferrara, Corso Ercole I d'Este 32,

44121 Ferrara, Italy

2 TekneHub, Tecnopolo of Ferrara University, Via Saragat 13,

44122 Ferrara, Italy 